\title{
«Ärzte und Patienten profitieren von der Vernetzung»
}

Guter Tradition folgend treffen sich die Schweizer eHealth-Interessierten am 22. und

23. September 2010 zu dem nationalen Kongress eHealthCare.ch. Für Dr. med. Urs Stoffel, Co-Präsident der Konferenz der Kantonalen ÄrzteGesellschaften, liegen in der Schweiz beim Thema eHealth Licht und Schatten eng beieinander.

Interview:

Philipp Grätzel von Grätz HealthTech Wire*

\footnotetext{
* HealthTech Wire ist der Nachrichtendienst des Kongresses eHealthCare.ch. Weitere Informationen unter www.healthtechwire.ch
}

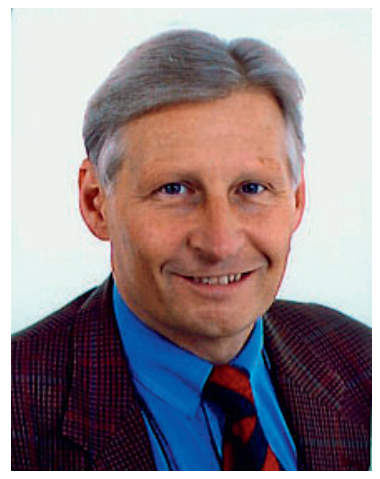

Urs Stoffel:

«Wir können jetzt Zahlen, mit denen die Kostenträger in Verhandlungen gehen, anhand eigener Daten plausibilisieren.»

Die Konferenz eHealthCare.ch jährt sich in diesem Jahr zum zehnten Mal. Wie weit ist die Schweiz bei der Umsetzung von eHealth-Anwendungen?

Urs Stoffel: Wir sind in einigen Segmenten des eHealth-Spektrums bereits recht gut aufgestellt, vor allem auch auf ärztlicher Seite. Insgesamt müssen wir aber schon konstatieren, dass die Schweiz in Sachen eHealth noch einen deutlichen Nachholbedarf hat. Die wichtigsten Akteure im Gesundheitswesen sind sich der Bedeutung von eHealth bewusst. An der ärztlichen Basis angekommen ist dieses Bewusstsein allerdings noch nicht überall. Auch deswegen sind Veranstaltungen wie eHealthCare.ch so wichtig.

Sie halten bei der Konferenz einen Vortrag zum Nutzen von Trust-Centern für die Ärzteschaft und für die Patienten. Weiss der durchschnittliche Schweizer Arzt, was ein Trust-Center ist?

Das ist einer der Punkte, an dem wir in der Schweiz schon relativ weit sind. Wir haben formal unabhängige, aber sehr ärzteschaftnahe Trust-Center vor einigen Jahren eingeführt, um die Abrechnungsdaten der Ärzte unter ärztlicher Hoheit elektronisch zusammenzuführen. Das wird mittlerweile sehr gut angenommen. Derzeit sind knapp 10000 der 14000 niedergelassenen Ärzte bei einem der zehn regionalen TrustCenter gemeldet, und das auf vollständig freiwilliger Basis. Um Ihre Frage zu beantworten: Ja, die Schweizer Ärzte wissen, was Trust-Center sind.
Worin bestehen die Vorteile dieser Konstruktion für die Ärzte?

Über die Trust-Center wurden mittlerweile nahezu eine Milliarde Datensätze eingesammelt, mit denen sehr viele sinnvolle Analysen gemacht werden können. Zum einen verfügt die Ärzteschaft damit erstmals über einen Datenfundus, der dem der Krankenversicherungsseite ebenbürtig ist. Wir können jetzt Zahlen, mit denen die Kostenträger in Verhandlungen gehen, anhand eigener Daten plausibilisieren. Die Position der Ärzte wird also gestärkt. Für den einzelnen Arzt können die gesammelten Daten zum Beispiel im Rahmen von Wirtschaftlichkeitsverfahren hilfreich sein, denn damit lassen sich viele Vorwürfe über unwirtschaftliches Handeln entkräften. Unabhängig davon können wir mit den Daten der TrustCenter auch aussagekräftige Versorgungsforschung betreiben, weil wir über einen nahezu flächendeckenden Datenschatz verfügen.

Wie andere europäische Länder plant auch die Schweiz den Aufbau einer nationalen eHealth-Infrastruktur auf Basis einer chipkartenbasierten Identifikation. Wird dieses Konzept von der Ärzteschaft mitgetragen?

\section{«Ja, die Schweizer Ärzte wissen, was Trust-Center sind»}

Grundsätzlich tun sich andere eHealth-Projekte in der Schweiz deutlich schwerer als die ärztlichen TrustCenter. Die Skepsis ist noch hoch, und es gibt eine Reihe ungelöster Fragen, etwa die nach einheitlichen Standards oder auch die Finanzierung der Investitionen. Das gilt für Projekte wie den Aufbau elektronischer Krankengeschichten und eben auch für den Aufbau der nationalen Infrastruktur. Zur Zeit wird die Ärzteschaft mit elektronischen Arztausweisen, den Health Professional Cards (HPC), ausgestattet. Auf Patientenseite haben wir mittlerweile eine Versicher- 
tenkarte eingeführt, die mittelfristig in Richtung einer elektronischen Gesundheitskarte weiter entwickelt werden soll. Viele Ärzte sehen das alles eher skeptisch. Ich selbst bin der Auffassung, dass viele Dinge auch ohne Karten sinnvoller und zielführender umgesetzt werden könnten, wenn dem Datenschutz mit anderen Mitteln Genüge getan wird. Wir haben beispielsweise mit dem HIN ein ärztliches Kommunikationsnetz, bei dem 11000 Ärzte und über 100 Spitäler

\section{«lch selbst bin der Auffassung, dass viele Dinge auch ohne Karten sinnvoller und zielführender umgesetzt werden könnten»}

angemeldet sind. Es nutzt spezielle Sicherheitssoftware. Das funktioniert wunderbar. Grundsätzlich kann man Chipkarten aber durchaus auf die existierenden Systeme aufsetzen. Es ist zum Beispiel denkbar, für die Datenübertragung zu den Trust-Centern künftig auch die HPC zu nutzen.

Wo sehen Sie auf Patientenseite den grössten Nutzen von eHealth-Anwendungen?

Ich denke schon, dass sich durch die elektronische Vernetzung die in vielen Fällen unbestreitbare Doppelspurigkeit der derzeitigen Versorgung verbessern lässt.
Durch die Einführung elektronischer Rezepte erhöht sich die Medikationssicherheit für die Patienten. Die Interaktion mit dem Gesundheitssystem wird bequemer, denken Sie nur an elektronische Terminbuchungen. Und natürlich beobachten wir in der Schweiz wie überall sonst auch einen Trend zu einer immer stärkeren Integration der Versorgungsketten, wodurch die Behandlung optimiert und gestrafft werden soll. Das funktioniert auf Dauer nur, wenn die Daten elektronisch fliessen. Im Moment haben wir das Problem, dass die zahlreichen Vorteile, die eHealth-Technologien bringen, noch nicht wirklich an der Basis angekommen sind. Deswegen brauchen wir Konferenzen wie die eHealthCare.ch, wo in zahlreichen Workshops und Kolloquien explizit aufgezeigt wird, wie Ärzte und Patienten von der Vernetzung profitieren können.

10. Schweizerischer eHealthcare-Kongress, Mittwoch und Donnerstag, 22./23. September 2010, GZI Seminar- und Kongresshotel, Nottwil LU

An diesem Jubiläums-Kongress wird Dr. med. Urs Stoffel ein Referat über das Thema «Trust Centers: Welchen Nutzen für die Ärzteschaft und welchen für die Patienten?» halten. Die Schweizerische Ärztezeitung ist Leading-Medienpartner des Kongress eHealthCare.ch. Das Konferenzprogramm und alle weiteren Informationen finden Sie online unter www.ehealthcare.ch

\section{Sie lesen gerade eine Zeitschrift des Schweizerischen Ärzteverlags EMH ...}

\section{Wussten Sie schon,}

- dass EMH ein Gemeinschaftsunternehmen der Verbindung der Schweizer Ärztinnen und Ärzte FMH und der Schwabe AG ist, dem mit Gründung 1488 ältesten Druck- und Verlagshaus der Welt?

- dass EMH mit insgesamt zehn Fachzeitschriften, einem umfangreichen Online-Angebot sowie einem wachsenden Buchprogramm der führende Verlag für medizinische Zeitschriften in der Schweiz ist?

n dass sämtliche bei EMH erscheinenden Zeitschriften offizielle Publikationsorgane der jeweils zuständigen medizinischen Fachorganisationen sind?

Wenn Sie mehr über EMH wissen möchten, finden Sie unter www.emh.ch weitere Informationen. 